\title{
SYSTEMS CHEMISTRY
}

\section{All in a spin}

A fundamental challenge in supramolecular systems chemistry is to engineer the emergence of complex behaviour. The collective structures of metal cyanide chains have now been interpreted in the same manner as the myriad of magnetic phases displayed by frustrated spin systems, highlighting a symbiotic approach between systems chemistry and magnetism.

\section{Lucy Clark and Philip Lightfoot}

Emergence is a subtle concept that is employed in a diverse range of scientific disciplines to describe an enormous variety of fascinating phenomena. An emergent property is one that arises in a system comprised of many interacting components, which we would not have otherwise expected, or predicted, based on our pre-existing knowledge of the individual components themselves. ${ }^{1}$ Arguably, the most important example of emergence is the origin of life on Earth. How was it that assemblies of non-living, simple chemical species that existed on prebiotic Earth crossed the boundary of life to allow living cells to emerge? ${ }^{2}$

Systems chemistry - the study of supramolecular assemblies and the nature of the interactions within them - is one field of chemical research that seeks to answer this fundamental question. ${ }^{3}$ A grand challenge of systems chemistry is to develop "bottom-up" methods to systematically engineer supramolecular ensembles of simple molecules from which specific, targeted and externally controllable functionality can emerge, ${ }^{4}$ such as the creation of artificial living cells. ${ }^{5}$ Now, writing in Nature Chemistry, a team led by Andrew Goodwin and François-Xavier Coudert has pioneered a novel approach towards the understanding and control of emergent behaviour by carving out a conceptual mapping between the collective structural behaviour of supramolecular assemblies of coordination polymer chains and the ostensibly unrelated field of frustrated magnetism. ${ }^{6}$ 
Frustrated magnetism continues to thrive as a highly popular, active area of research within the condensed matter physics community, not least because frustrated magnets themselves display a particularly impressive set of emergent physical phenomena. Magnetic frustration can occur when the arrangement of individual magnetic moments - often referred to as spins - obstructs the dominant magnetic exchange interactions between neighbouring spins from taking place. In the absence of frustration, the magnetic exchange interactions between spins tend to promote the formation of simple, long-range magnetic order with neighbouring spins, aligned either in the same direction, in a ferromagnet (Fig. 1a), or in an alternately opposed fashion, in an antiferromagnet (Fig. 1b). A canonical example of a system that is magnetically frustrated is the triangular XY magnet, to which Goodwin, Coudert and coworkers refer in their work.

In this particular magnetic model, spins are constrained to lie within a single plane and are arranged on a two-dimensional triangular lattice made up of equilateral triangles that share edges. If the dominant magnetic exchange interaction between neighbouring spins is antiferromagnetic it is not possible to satisfy this pair-wise interaction between all spins simultaneously, thus the system is frustrated (Fig. 1c). As the size of the system grows from a single triangular plaquette of spins to an infinite triangular lattice, this frustration promotes highly unconventional magnetic behaviour, and exotic emergent phenomena abound. ${ }^{7}$

In the present study, the researchers noted that in frustrated magnetism the understanding, and prediction, of the emergent behaviour of a particular system is often made somewhat simpler through judicious control over the geometry and type of its magnetic exchange interactions. They then sought to make use of this approach in order to decipher the complexity that can be displayed in supramolecular systems chemistry. Their study focuses on supramolecular assemblies of non-magnetic gold and silver cyanide polymer chains. The relevant supramolecular interactions within these two systems are either electrostatic or metallophilic in nature and their assemblies are made up of linear $-\mathrm{M}-\mathrm{CN}-\mathrm{M}-$ chains, where $\mathrm{M}=\mathrm{Au}$ or $\mathrm{Ag}$, which are arranged onto a triangular lattice. The difference between the $\mathrm{AuCN}$ and $\mathrm{AgCN}$ polymer assemblies is that in the case of the AuCN system, neighbouring chains are aligned such that each $\mathrm{Au}^{+}$cation is aligned with other $\mathrm{Au}^{+}$cations, where as in the $\mathrm{AgCN}$ system, there is a continuous displacement of neighbouring chains, such that each $\mathrm{Ag}^{+}$ cation is aligned with $\mathrm{CN}^{-}$anions.

To understand the difference in the collective structural behaviour of these two supramolecular assemblies, the authors have established a mapping between the structures of the two polymer systems and the spin structures of triangular XY magnets by demonstrating the mathematical equivalence between the relative displacement of the neighbouring polymer chains in the supramolecular system and an angle that corresponds to the relative orientation of neighbouring spins on a triangular magnetic lattice (Fig. 1d). In the case of $\mathrm{AgCN}$, this mapping leads to an antiferromagnetic spin configuration on the triangular lattice, whereas the AuCN structure maps to the triangular ferromagnet.

Through quantum mechanical calculations the researchers show that the collective structures of the AuCN and AgCN polymer assemblies are a direct result of their supramolecular self- 
interaction potential energies. For $\mathrm{AuCN}$ the dominant interaction between chains is the metallophilic one, which favours the close metal-metal contacts, whereas for $\mathrm{AgCN}$ it is the electrostatic interactions that dominate, leading to the displacement of neighbouring chains to exactly avoid those metal-metal contacts. An important point noted by the researchers is that the distinct supramolecular self-interaction potentials of the two different polymer chain assemblies are well approximated by a simple Fourier series expansion. This allowed them to demonstrate that the mapping to the different phases of the triangular $\mathrm{XY}$ magnet is no coincidence, and that the supramolecular interaction potentials of the $\mathrm{AgCN}$ and $\mathrm{AuCN}$ assemblies directly correspond to antiferro- and ferromagnetic nearest-neighbour exchange energies, respectively.

As pointed out by the researchers, this is not the first example of a conceptual mapping between purely structural and complex magnetic systems in frustrated magnetism. ${ }^{8}$ However, what is remarkable in this instance is that the use of the simple mapping developed for the $\mathrm{AuCN}$ and $\mathrm{AgCN}$ polymer systems to begin to understand the altogether more complicated collective behaviour of supramolecular assemblies of bimetallic gold and silver cyanide, the true structure of which has eluded us up until now. The bimetallicity introduces a greater complexity within the overall system by allowing for both heterophilic and homophilic metal-metal interactions. The determination of the form of the supramolecular interaction potentials in the $\mathrm{Au}_{0.5} \mathrm{Ag}_{0.5} \mathrm{CN}$ assembly, and hence a suitable mapping to a triangular $\mathrm{XY}$ magnet, allowed the structure of the bimetallic system to be elucidated from its experimental powder X-ray diffraction data.

Particularly exciting is the observation that the magnetic phase obtained from the mapping of the bimetallic supramolecular interaction potential corresponds to an unconventional spin nematic state, in which the spins align along a preferred axis without demonstrating any long range order of the absolute spin direction along that axis. This type of magnetic order has been theoretically predicted for XY spins on a triangular lattice, but experimental realisations of such a state are yet to be found. To fully account for the experimental data on $\mathrm{Au}_{0.5} \mathrm{Ag}_{0.5} \mathrm{CN}$, the researchers found that it was necessary to include thermal effects within their model, which in the case of the mapped spin triangular XY system corresponds to the presence of spin vortices that emerge from the nematic ground state at finite temperatures. Systems that can play host to such spin vortices - a type of collective magnetic structure that exhibits an in-plane curling of spins - are attracting considerable attention as potential data storage devices. ${ }^{9}$

The significance of this study is, therefore, two-fold. First, the clear demonstration that the emergence of a variety of different supramolecular polymer structures is driven by their chemically tuneable interaction potentials, and that the nature of these structures can be reliably predicted through a mapping to different phases of frustrated magnets. Second, the fact that that through this mapping these supramolecular assemblies act as structural analogues of highly complex magnetic states of matter that are otherwise yet to be discovered. There is great scope to explore how the collective structures of the bimetallic polymer assemblies respond to external perturbations, including changes in temperature, pressure and chemical substitution. By determining the variations in the ensuing 
supramolecular interaction potentials, one could map out the corresponding variations of the magnetic exchange interactions within the analogous magnetic phases. Compellingly, this may reveal the types of emergent phenomena we can expect to observe in frustrated magnetic systems that are currently out of our experimental reach. Finally, it is important to emphasise the truly symbiotic nature of this study. Not only will it be of great potential benefit to supramolecular systems chemists who may seek to employ this approach to allow for the informed design of assemblies with specific emergent properties, but undoubtedly, it will also be enormously beneficial to hard condensed matter physicists, who are always driven to uncover new ways to reveal the collective emergent behaviours of the most fascinating theoretically-predicted phases of frustrated magnets. One could even argue - given the seemingly unrelated nature of these two fields of up until now - that the consequently unexpected mutually-beneficial relationship discovered between them here is a prime example of emergence in and of itself!

Acknowledgement: We thank the Leverhulme Trust for an award (RPG-2013-343) to support LC.

\title{
References:
}

${ }^{1}$ Balazs, A. C. \& Epstein, I. R. Science 325, 1632-1634 (2009).

${ }^{2}$ Rasmussen, S. et al. Science 303, 963-965 (2004).

${ }^{3}$ Mattia, E. \& Otto, S. Nature Nanotech. 10, 111-119 (2015).

${ }^{4}$ Nitschke, J. R. Nature 462, 736-738 (2009).

${ }^{5}$ Szostak, J. W., Bartel, D. P. \& Luisi, P. L. Nature 409, 387-309 (2001).

${ }^{6}$ Cairns, A. B. et al. Nature Chem., in press (2016).

${ }^{7}$ Lee, S. - H. et al. Nature 418, 856-858.

${ }^{8}$ Harris, M. J., Bramwell, S. T., McMorrow, D. F., Zeiske, T. \& Godfrey, K. W. Phys. Rev. Lett. 79, 2554-2557 (1997).

${ }^{9}$ Mühlbauer, S. et al. Science 323, 915-919 (2009).

\author{
Authors' affiliations \\ $<$ School of Chemistry and EaStChem, University of St Andrews, St Andrews, Fife, \\ KY16 9ST, U.K. e-mail pl@st-and.ac.uk>
}

Figure 1. Schematic representation of frustrated magnets and the mapping of assemblies of coordination polymer chains in supramolecular systems chemistry. a-d, 
The simple magnetic structures obtained from the ferromagnetic (a) and antiferromagnetic (b) ordering of neighbouring spins. When spins are arranged on an equilateral triangular plaquette (c), simple antiferromagnetic ordering is frustrated as a consequence of their relative geometry (left). However, if the spins are allowed to freely rotate in the plane (i.e. $\mathrm{XY}$ spins) they can adopt a compromise spin configuration to alleviate the effects of frustration (right). (d), In the supramolecular polymer assemblies of gold and silver cyanide, the polymer chains are packed onto a triangular lattice of edge sharing equilateral triangles. In the case of the $\mathrm{AgCN}$ assembly, the specific supramolecular interactions between the chains results in their staggered vertical displacement, $z$ (top). Mapping this vertical displacement onto a mathematically equivalent phase angle gives rise to three distinct spin orientations and the triangular XY antiferromagnet (bottom).

(a)

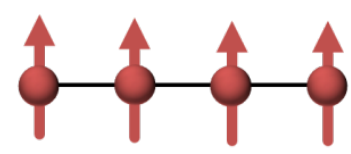

(d)

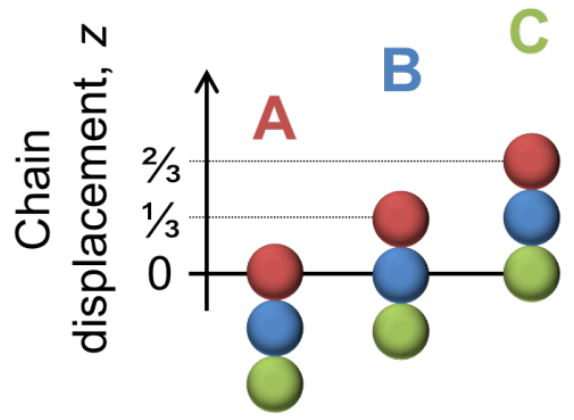

(b)

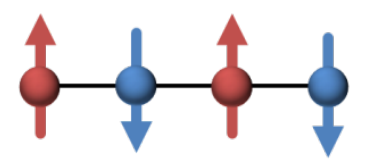

8 = a polymer chain

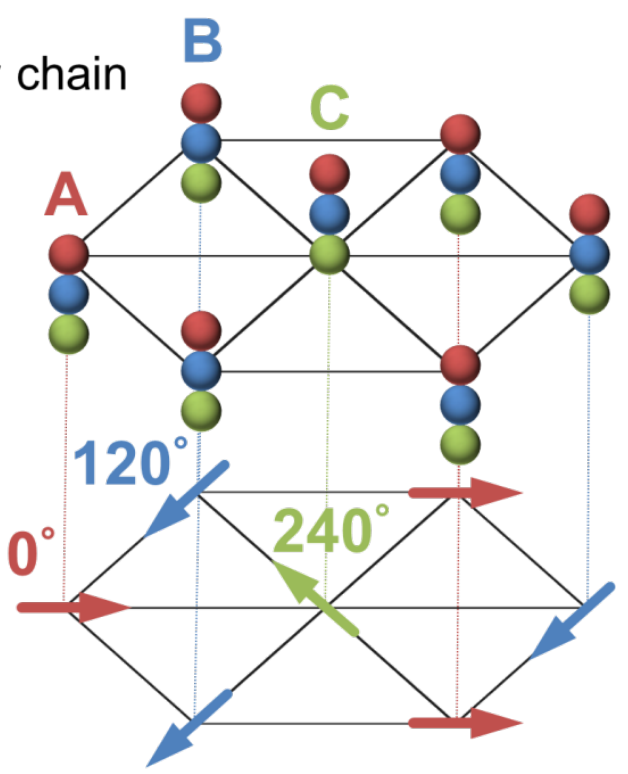

